OPEN ACCESS

Edited by:

Alejandro Gru,

University of Virginia Health System,

United States

Reviewed by:

Deepshi Thakral,

All India Institute of Medical

Sciences, India

Michael Diamantidis,

University Hospital of Larissa, Greece

${ }^{*}$ Correspondence:

Li Chai

Ichai@bwh.harvard.edu

Lu-Hong Xu

xulhong@mail.sysu.edu.cn

Specialty section: This article was submitted to Hematologic Malignancies,

a section of the journa

Frontiers in Oncology

Received: 22 November 2020 Accepted: 29 March 2021

Published: 21 April 2021

Citation:

Wang $Y$, Weng W-J, Zhou D-H, Fang J-P, Mishra S, Chai L and XU L-H (2021) Wilms Tumor 1 Mutations Are Independent Poor Prognostic Factors in Pediatric Acute Myeloid Leukemia.

Front. Oncol. 11:632094.

doi: 10.3389/fonc.2021.632094

\section{Wilms Tumor 1 Mutations Are Independent Poor Prognostic Factors in Pediatric Acute Myeloid Leukemia}

\author{
Yin Wang ${ }^{1,2}$, Wen-Jun Weng ${ }^{1,2}$, Dun-Hua Zhou ${ }^{1,2}$, Jian-Pei Fang ${ }^{1,2}$, Srishti Mishra ${ }^{3}$, \\ Li Chai ${ }^{4 *}$ and Lu-Hong $\mathrm{Xu}^{1,2 *}$ \\ 1 Department of Pediatrics, Sun Yat-sen Memorial Hospital, Sun Yat-sen University, Guangzhou, China, ${ }^{2}$ Guangdong \\ Provincial Key Laboratory of Malignant Tumor Epigenetics and Gene Regulation, Sun Yat-sen Memorial Hospital, \\ Sun Yat-sen University, Guangzhou, China, ${ }^{3}$ Cancer Science Institute, Yong Loo Lin School of Medicine, National University \\ of Singapore, Singapore, Singapore, ${ }^{4}$ Department of Pathology, Brigham \& Women's Hospital, Harvard Medical School, \\ Boston, MA, United States
}

The prognostic impact of Wilms tumor 1 (WT1) mutations remains controversial for patients with acute myeloid leukemia (AML). Here, we aimed to determine the clinical implication of WT1 mutations in a large cohort of pediatric AML. The clinical data of 870 pediatric patients with $A M L$ were downloaded from the therapeutically applicable research to generate effective treatment (TARGET) dataset. We analyzed the prevalence, clinical profile, and prognosis of AML patients with WT1 mutations in this cohort. Our results showed that $6.7 \%$ of total patients harbored WT1 mutations. These WT1 mutations were closely associated with normal cytogenetics $(P<0.001)$, FMS-like tyrosine kinase $3 /$ internal tandem duplication (FLT3/ITD) mutations $(P<0.001)$, and low complete remission induction rates $(P<0.01)$. Compared to the patients without WT1 mutations, patients with WT1 mutations had a worse 5-year event-free survival $(21.7 \pm 5.5 \%$ vs $48.9 \pm 1.8 \%$, $P<0.001)$ and a worse overall survival $(41.4 \pm 6.6 \%$ vs $64.3 \pm 1.7 \%, P<0.001)$. Moreover, patients with both WT1 and FLT3/ITD mutations had a dismal prognosis. Compared to chemotherapy alone, hematopoietic stem cell transplantation tended to improve the prognoses of WT1-mutated patients. Multivariate analysis demonstrated that WT1 mutations conferred an independent adverse impact on event-free survival (hazard ratio 1.910, $P=0.001$ ) and overall survival (hazard ratio 1.709, $P=0.020$ ). In conclusion, our findings have demonstrated that WT1 mutations are independent poor prognostic factors in pediatric AML.

Keywords: acute myeloid leukemia, WT1 mutations, pediatric patients, prognostic factors, FLT3/ITD mutations

\section{INTRODUCTION}

Acute myeloid leukemia (AML) is a type of blood cancer that originates in the bone marrow from immature white blood cells known as myeloblasts. About $20 \%$ of all children with leukemia have AML $(1,2)$. In the last few years, collaborative studies have revealed a link between the degree of genetic heterogeneity of AML and the clinical outcome, allowing risk stratification before therapy 
and guiding post-induction treatment (3). The Wilms tumor 1 (WT1) gene, located on chromosome 11p13, encodes a zincfinger protein that exists in multiple isoforms. It has been implicated in the regulation of cell survival, proliferation and differentiation, and may function both as a tumor suppressor and an oncogene $(4,5)$. Various mutations across WT1 gene have been reported in solid tumors and AML $(6,7)$. However, the prognostic impact of WT1 mutations remains controversial for patients with AML (8).

The WT1 mutations have been shown to be independent predictors of worse clinical outcome in some but not all adult AML studies (9-11). Recently, WT1 mutations are proposed to be prognostic markers of risk stratification for adult AML (12). However, the prognostic implications of WT1 mutations have not been clarified in pediatric AML. Moreover, large cohort studies on the clinical significance of WT1 mutations in pediatric AML are scarce. A pediatric study of 298 patients with AML found that WT1 mutations conferred an independent poor prognostic significance (13). However, another study of 842 pediatric AML revealed that the presence of WT1 mutations had no independent prognostic significance in predicting the disease outcome (14). Recently, in a cohort of 353 pediatric patients with AML, Niktoreh et al. (15) have found that WT1 mutations significantly increased the chance of relapse or treatment failure and reduced the probability of 3-year overall survival (OS), but had no significant impact on the 3-year probability of event-free survival (EFS). On the other hand, hematopoietic stem cell transplantation (HSCT) is an important treatment modality for patients with AML. However, the role of HSCT for patients with WT1 mutations remains unknown.

To determine the clinical implication of WT1 mutations, an independent large cohort study of pediatric AML is needed. Therefore, we analyzed the clinical data of 870 pediatric patients with AML from the therapeutically applicable research to generate effective treatment (TARGET) dataset. We found that WT1 mutations are independent poor prognostic factors in pediatric AML in terms of 5-year EFS and OS. Patients with both $W T 1$ and FMS-like tyrosine kinase 3/internal tandem duplication (FLT3/ITD) mutations had a dismal prognosis. Moreover, HSCT might be an effective strategy for patients with WT1 mutations.

\section{MATERIALS AND METHODS}

\section{Patients}

The clinical data on patients with AML were downloaded from the TARGET dataset (https://ocg.cancer.gov/programs/target/ data-matrix). In total, 870 pediatric patients younger than 18 years old with the information of WT1 mutations were included in our study. The year of diagnosis ranged from 1996 to 2010 while the year of last follow-up ranged from 1997 to 2015. The diagnosis of pediatric AML and risk stratification were defined according to the Children's Oncology Group (COG) guidelines. Subtype classifications of AML were assigned according to the
French-American-British (FAB) classifications. Mutation analyses of WT1, FLT3/ITD, NPM1, and CEBPA were performed as previously described (14, 16-18). Treatment protocols for AML included AAML03P1, AAML0531 and CCG-2961. HSCT was considered for high-risk patients in the first complete remission. Detailed treatments and risk stratification of these studies have been previously described (19).

\section{Statistical Analysis}

The data were analyzed with the Statistical Package for the Social Sciences (SPSS ${ }^{\circledR}$ ) version, 20.0 (IBM Corporation, Armonk, NY, USA). The $\chi 2$ test was used to compare the frequencies of mutations. Fischer's exact test was used when data were sparse. The nonparametric Mann-Whitney $U$-test was applied for continuous variables. Complete remission (CR) was defined as bone marrow aspirate with $<5 \%$ blasts by morphology. EFS was defined as the time between diagnosis and first event, including induction failure, relapse, or death of any cause. OS was defined as the time between diagnosis and death from any cause. The survival curves were estimated using the Kaplan-Meier method and compared using the log-rank test. Cox proportional hazard models were used to estimate hazard ratios (HR) for multivariate analyses. A two-sided $P$-value less than 0.05 was considered statistically significant for all statistical analyses.

\section{RESULTS}

\section{Relationship Between WT1 Mutations and Clinical Characteristics}

The patients' clinical characteristics are shown in Table 1. Overall, among the 870 pediatric patients with AML, 58 patients $(6.7 \%)$ were identified with WT1 mutations. The white blood cell count (WBC) at diagnosis was significantly higher in WT1-mutated patients (median $56.9 \times 10^{9} / \mathrm{L}$ ) than in $W T 1$ wild-type patients (median $30.8 \times 10^{9} / \mathrm{L} ; P=0.041$ ). In $W T 1$-mutated group, the FAB subtypes were mainly M1, M2, and M4. A higher proportion of WT1-mutated patients had M4 morphology in comparison with WT1 wild-type patients $(41.2 \%$ vs $25.9 \% ; P=0.018)$. We also evaluated the associations between WT1 mutations and cytogenetic and molecular alterations. In terms of cytogenetics, WT1 mutations were found more frequently in the normal cytogenetics subset (44.2\% of WT1-mutated patients had normal cytogenetics compared with $22.3 \%$ of those without WT1 mutations; $P<0.001)$. Regarding the molecular alterations, there was a substantial overlap between WT1 mutations and FLT3/ITD, as shown in Table 1, 48.3\% of those carrying a WT1 mutation were also FLT3/ITD positive as opposed to $14.7 \%$ of patients without WT1 mutations $(P<0.001)$. Moreover, the WT1-mutated patients were classified more frequently as high risk $(40.7 \%$ vs $12.6 \% ; P<0.001)$. The treatment protocols for pediatric AML were equally distributed between these two groups $(P=0.058)$. However, there were no significant differences in the median age, the median of FLT3/ITD allelic ratio, NPM1, and CEBPA mutations between the WT1-mutated group and WT1 wild-type group. 
TABLE 1 | Characteristics of pediatric patients with or without WT1 mutations.

\begin{tabular}{|c|c|c|c|c|}
\hline & All patients & WT1-mutated case & WT1 wildtype case & $P$-value \\
\hline Number (\%) & 870 & $58(6.7 \%)$ & 812(93.3\%) & \\
\hline Age, median (year) & 9.6 & 11 & 9.5 & 0.221 \\
\hline$<3$ years, n (\%) & $211(24.3 \%)$ & $6(10.3 \%)$ & 205 (25.2\%) & 0.011 \\
\hline $3 \leq$ Age<10years, n (\%) & $237(27.2 \%)$ & 19 (32.8\%) & $218(26.8 \%)$ & 0.329 \\
\hline $10 \leq$ Age<18years, n (\%) & $422(48.5 \%)$ & $33(56.9 \%)$ & $389(47.9 \%)$ & 0.186 \\
\hline Sex & & & & 0.119 \\
\hline male, n (\%) & 454 (52.2\%) & $36(62.1 \%)$ & $418(51.5 \%)$ & \\
\hline female, n (\%) & $416(47.8 \%)$ & $22(37.9 \%)$ & 394 (48.5\%) & \\
\hline \multicolumn{5}{|l|}{ WBC, $\times 10^{9} / \mathrm{L}$} \\
\hline Median (range) & $31.7(0.2-610)$ & $56.9(1.1-446)$ & $30.8(0.2-610)$ & 0.041 \\
\hline FAB classification: n (\%) & & & & 0.001 \\
\hline MO & $20(2.8 \%)$ & $1(2.0 \%)$ & 19 (2.9\%) & $>0.999$ \\
\hline M1 & 96 (13.4\%) & 10 (19.6\%) & $86(13.0 \%)$ & 0.181 \\
\hline M2 & $193(27.0 \%)$ & $11(21.6 \%)$ & $182(27.5 \%)$ & 0.362 \\
\hline M3 & $2(0.3 \%)$ & $0(0.0 \%)$ & $2(0.3 \%)$ & $>0.999$ \\
\hline M4 & $193(27.0 \%)$ & $21(41.2 \%)$ & $172(25.9 \%)$ & 0.018 \\
\hline M5 & 160 (22.4\%) & $3(5.9 \%)$ & 157 (23.7\%) & 0.003 \\
\hline M6 & $11(1.5 \%)$ & $4(7.8 \%)$ & $7(1.1 \%)$ & 0.005 \\
\hline $\mathrm{M} 7$ & $39(5.5 \%)$ & $1(2.0 \%)$ & $38(5.7 \%)$ & 0.351 \\
\hline Risk group: n (\%) & & & & $<0.001$ \\
\hline Low risk & 328 (39.0\%) & 15 (27.8\%) & 313 (39.8\%) & 0.079 \\
\hline Standard risk & 391 (46.5\%) & 17 (31.5\%) & 374 (47.6\%) & 0.022 \\
\hline High risk & $121(14.4 \%)$ & $22(40.7 \%)$ & 99 (12.6\%) & $<0.001$ \\
\hline FLT3/ITD & & & & $<0.001$ \\
\hline Positive, n (\%) & 147 (16.9\%) & 28 (48.3\%) & 119 (14.7\%) & \\
\hline Negative, n (\%) & 722(83.1\%) & 30 (51.7\%) & 692 (85.3\%) & \\
\hline FLT3/ITD allelic ratio, & 0.54 & 0.55 & 0.54 & 0.865 \\
\hline Median (range) & $(0.03-9.50)$ & $(0.03-5.19)$ & $(0.03-9.50)$ & \\
\hline NPM1 & & & & 0.794 \\
\hline Positive, n (\%) & $66(7.6 \%)$ & $3(5.3 \%)$ & $63(7.8 \%)$ & \\
\hline Negative, n (\%) & 802(92.4\%) & 63(94.7\%) & $748(92.2 \%)$ & \\
\hline CEBPA & & & & 0.245 \\
\hline Positive, n (\%) & $49(5.7 \%)$ & $1(1.7 \%)$ & $48(5.9 \%)$ & \\
\hline Negative, n (\%) & $817(94.3 \%)$ & 57(98.3) & 760(94.1\%) & \\
\hline \multicolumn{5}{|l|}{ Cytogenetic status } \\
\hline Normal (n, \%) & 196(23.7\%) & $23(44.2 \%)$ & 173(22.3\%) & $<0.001$ \\
\hline Abnormal (n, \%) & 631 (76.4\%) & 29 (55.8\%) & 602 (77.7\%) & 0.317 \\
\hline $\operatorname{inv}(16)(n, \%)$ & $106(12.8 \%)$ & $9(17.3 \%)$ & $97(12.5 \%)$ & 0.046 \\
\hline $\mathrm{t}(8 ; 21)(\mathrm{n}, \%)$ & $128(15.5 \%)$ & $3(5.8 \%)$ & $125(16.1 \%)$ & \\
\hline HSCT in 1st CR & & & & 0.906 \\
\hline No (n, \%) & $663(83.8 \%)$ & 38 (84.4\%) & $625(83.8 \%)$ & \\
\hline Yes (n, \%) & 128 (16.2\%) & 7 (15.6\%) & 121 (16.2\%) & \\
\hline Protocol & & & & 0.058 \\
\hline AAML03P1 (n, \%) & $91(10.5 \%)$ & 7 (12.1\%) & 84 (10.3\%) & 0.679 \\
\hline AAML0531 (n, \%) & $732(84.1 \%)$ & $44(75.9 \%)$ & $688(84.7 \%)$ & 0.074 \\
\hline CCG-2961 (n, \%) & $47(5.4 \%)$ & 7 (12.1\%) & $40(4.9 \%)$ & 0.031 \\
\hline CR status at end of course 1 & & & & 0.002 \\
\hline CR, n (\%) & $656(76.3 \%)$ & 35 (60.3\%) & $621(77.4 \%)$ & 0.003 \\
\hline Not CR, n (\%) & $189(22.0 \%)$ & $20(34.5 \%)$ & $169(21.1 \%)$ & 0.017 \\
\hline Death, n (\%) & $15(1.7 \%)$ & $3(5.2 \%)$ & $12(1.5 \%)$ & 0.074 \\
\hline CR status at end of course 2 & & & & $<0.001$ \\
\hline CR, n (\%) & $736(87.2 \%)$ & $38(69.1 \%)$ & $698(88.5 \%)$ & $<0.001$ \\
\hline Not CR, n (\%) & $88(10.4 \%)$ & $14(25.5 \%)$ & 74 (9.4\%) & $<0.001$ \\
\hline Death, n (\%) & $20(2.4 \%)$ & $3(5.5 \%)$ & $17(2.2 \%)$ & 0.136 \\
\hline
\end{tabular}

CEBPA CCAAT, enhancer binding protein alpha; CR, complete remission; FAB, French-American-British morphology classification; FLT3/ITD, internal tandem duplication of the FLT3 gene; HSCT, hematopoietic stem cell transplantation; NPM1, Nucleophosmin; WBC, white blood cell count.

\section{Clinical Outcome and Prognostic Effect of WT1 Mutations}

The CR rate was determined for all patients after the first and second course of induction therapy. At the end of the first course of therapy, patients with WT1 mutations had a lower rate of CR (60.3\%) compared to those without WT1 mutations (77.4\%), and the difference was statistically significant $(P=0.002)$. At the end of the second course of therapy, 38(69.1\%) of the 55 patients with WT1 mutations achieved a CR compared to 698 (88.5\%) of 789 patients without WT1 mutations $(P<0.001)$. Taken together, WT1 mutations were significantly associated with low induction $\mathrm{CR}$ rates. 
A

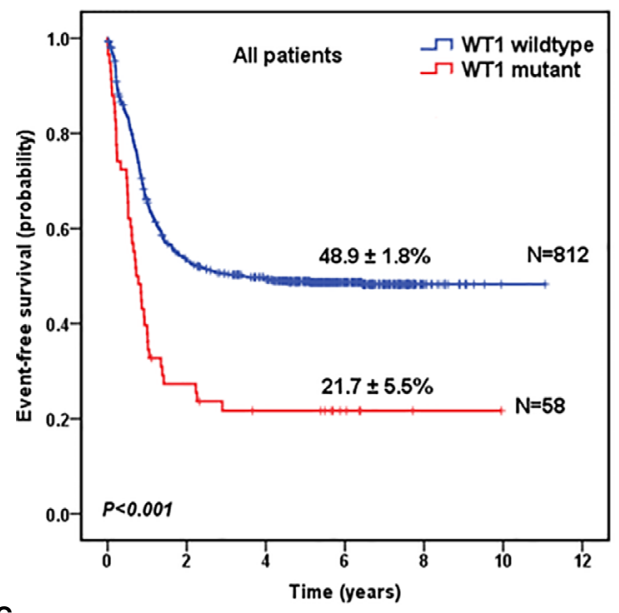

C

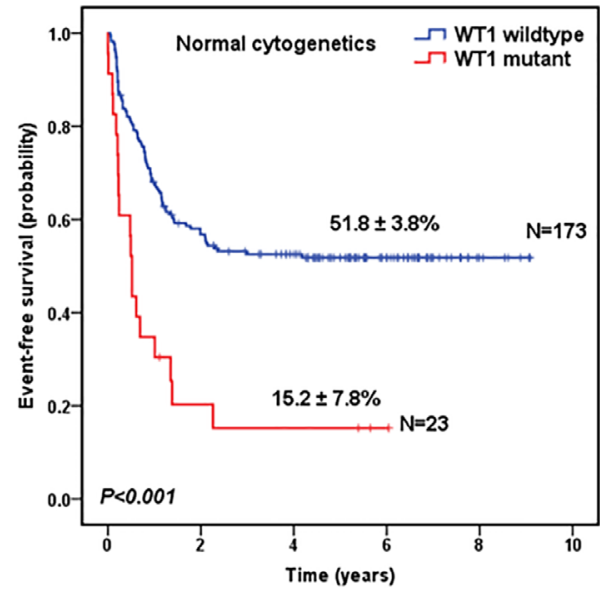

E

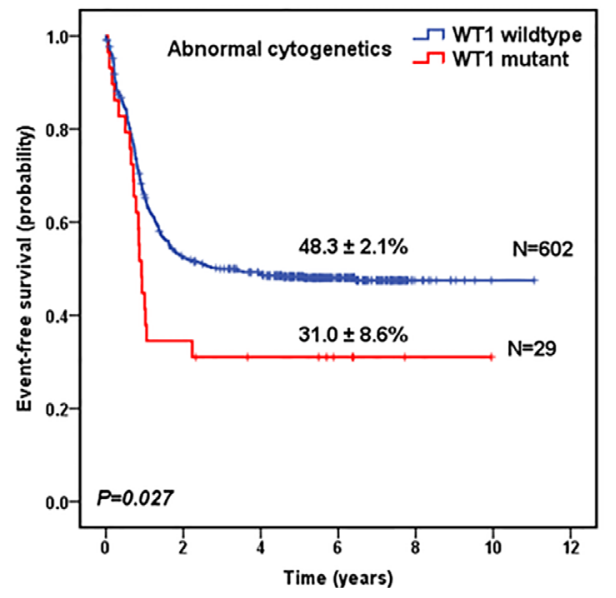

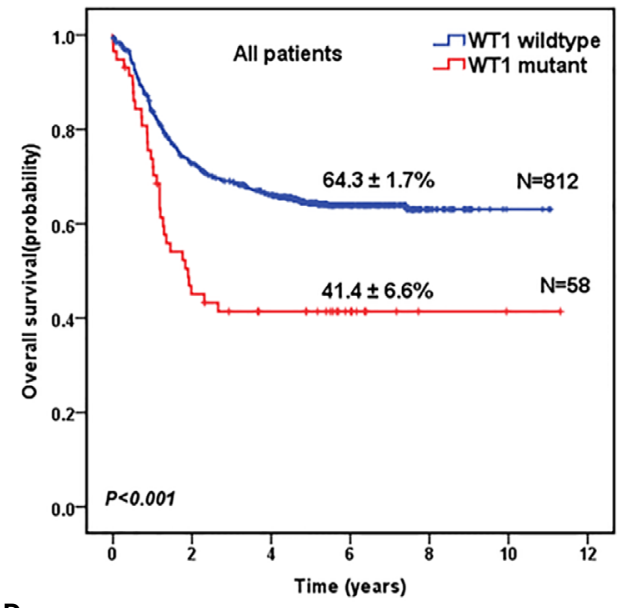

D
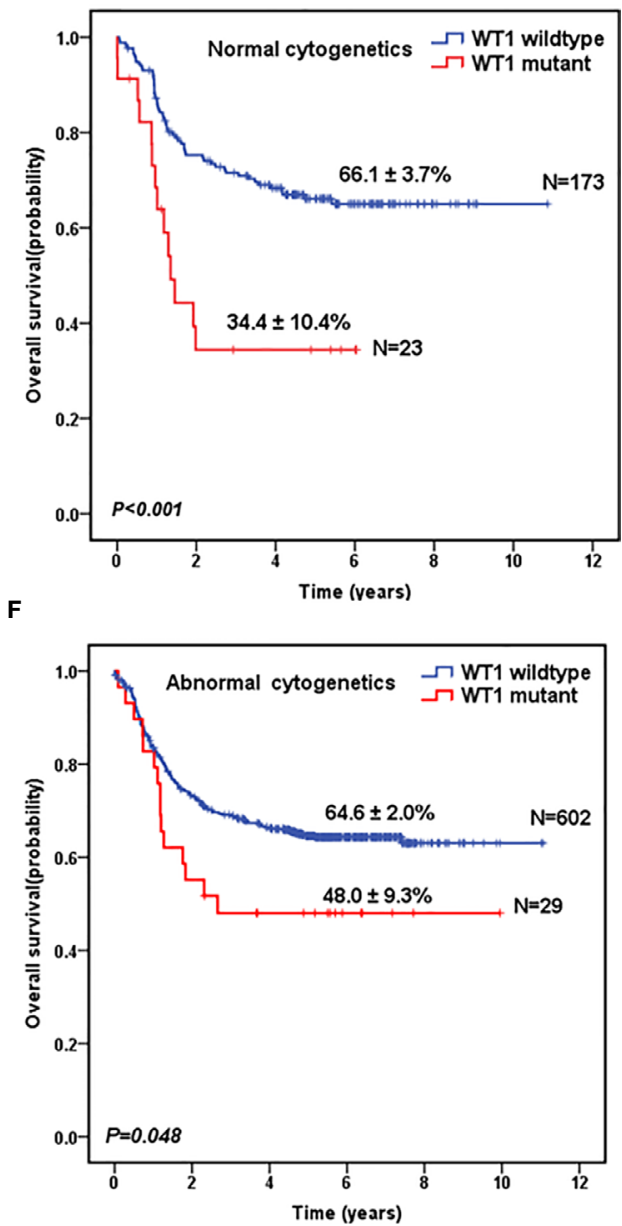

FIGURE 1 | Survival curves of pediatric AML patients with and without WT1 mutations. Probability of EFS (A) and OS (B) for all patients with and without WT1 mutations, respectively. Probability of EFS (C) and OS (D) for cytogenetically normal patients with and without WT1 mutations, respectively. Probability of EFS (E) and OS (F) for cytogenetically abnormal patients with and without WT1 mutations, respectively. 
Next, we evaluated the survival data for all the 870 pediatric patients. The median follow-up time for the survivors was 5.6 years. As shown in Figure 1A, WT1mutated patients had a significantly worse 5-year EFS $(21.7 \pm 5.5 \%)$ compared with WT1 wild-type patients $(48.9 \pm 1.8 \% ; P<0.001)$. Moreover, patients with WT1 mutations had a worse 5-year OS $(41.4 \pm 6.6 \%)$ than those without WT1 mutations $(64.3 \pm 1.7 \%$; $P<0.001)$ (Figure 1B). When analyses were restricted to patients having normal cytogenetics, there were significant differences in the outcome between patients with and without WT1 mutations (Figures 1C, D) (5-year EFS: $15.2 \pm 7.8 \%$ vs $51.8 \pm 3.8 \%$, $P<0.001$; 5 -year OS: $34.4 \pm 10.4 \%$ vs $66.1 \pm 3.7 \%, P<0.001)$. In the subgroup of abnormal cytogenetics (Figures 1E, F), WT1mutated patients also had a worse survival time compared with WT1 wild-type patients in terms of 5-year EFS (31.0 \pm $8.6 \%$ vs $48.3 \pm 2.1 \%, P=0.027)$ and $\mathrm{OS}(48.0 \pm 9.3 \%$ vs $64.6 \pm$ $2.0 \%, P=0.048)$.

\section{Prognostic Impact of WT1 and FLT3/ITD Mutations}

Survival data for patients with FLT3/ITD positive and negative were also explored. As shown in Figure S1A, FLT3/ITD positive was significantly associated with inferior EFS (5-year EFS $=33.5 \pm$ $4.0 \%$ vs $49.7 \pm 1.9 \%$ for $F L T 3 /$ ITD-negative; $P<0.001)$. Moreover, the FLT3/ITD positive group had a worse 5-year OS (51.5 \pm $4.3 \%)$ than the FLT3/ITD-negative group $(65.0 \pm 1.8 \% ; P=0.003)$

\section{(Figure S1B).}

Given the overlap between WT1 mutations and positive FLT3/ITD status, subset analysis was performed to assess the relative influence of WT1 mutations and FLT3/ITD on the prognosis of children with AML (Figures 2A, B; Table 2). In the FLT3/ITD-positive subgroup, WT1-mutated patients had an extremely dismal prognosis (5-year EFS $=12.5 \pm 6.5 \%$ vs $38.4 \pm$ $4.5 \%$ for WT1 wild-type patients, HR: 2.179 [1.364-3.482], $P=0.001$; 5 -year $\mathrm{OS}=27.5 \pm 8.8 \%$ vs $57.0 \pm 4.7 \%$ for $W T 1$ wild-type patients, HR: 2.225[1.305-3.796], $P=0.003)$. When
A

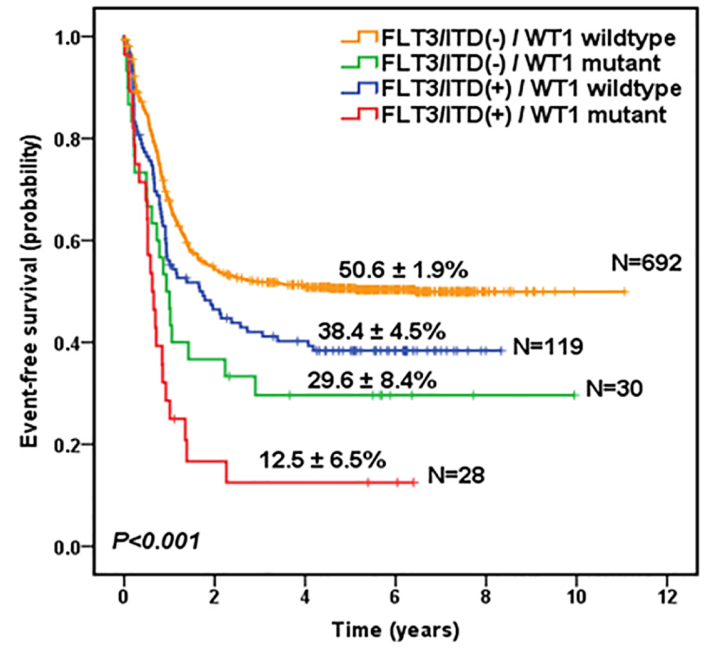

B

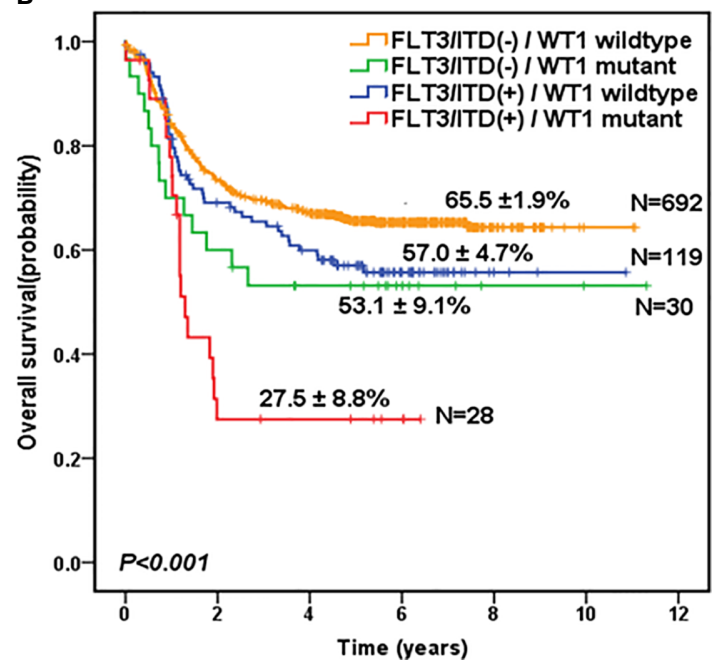

FIGURE 2 | Survival curves of all pediatric AML patients according to the combined WT1 mutations and positive FLT3/ITD status. Probability of EFS (A) and OS (B) for patients according to the combined WT1 mutations and positive FLT3/TTD status, respectively.

TABLE 2 | Statistical comparison of survival data according to both WT1 and FLT3/ITD status.

\begin{tabular}{lccc}
\hline Comparison & EFS hazard ratio (95\% Cl) & $\begin{array}{c}\text { EFS } \\
\text { P-value }\end{array}$ & $\begin{array}{c}\text { OS hazard ratio } \\
\text { (95\% Cl) }\end{array}$ \\
\hline $\begin{array}{l}\text { FLT3/ITD(-): } \\
\text { WT1 wildtype vs WT1 mutant }\end{array}$ & $1.861(1.197-2.892)$ & 0.006 & $1.600(0.933-2.744)$ \\
FLT3/ITD(+): & $2.179(1.364-3.482)$ & 0.001 & 0.088 \\
WT1 wildtype vs WT1 mutant & $1.386(1.075-1.788)$ & 0.012 & 0.003 \\
WT1 wildtype: & $1.605(0.886-2.906)$ & 0.118 & $1.305(0.961-1.771)$ \\
FLT3/ITD(-)vs FLT3/ITD(+) & & & $1.748(0.870-3.514)$ \\
WT1 mutant: & & & 0.088 \\
FLT3/ITD(-) vs FLT3/TD(+) & &
\end{tabular}

Cl, confidence interval; EFS, event-free survival; FLT3/ITD, internal tandem duplication of the FLT3 gene; OS, overall survival. 
restricted to the FLT3/ITD-negative subgroup, WT1 mutations had an adverse impact on 5-year EFS (HR: 1.861[1.197-2.892], $P=0.006$ ) instead of 5-year OS (HR: 1.600[0.933-2.744], $P=0.088)$. Similarly, for the WT1 wild-type patients, FLT3/ITD positive had reduced 5-year EFS (HR: 1.386[1.075-1.788], $P=0.012$ ) but not 5-year OS (HR: 1.305[0.961-1.771], $P=0.088$ ). However, FLT3/ITD mutations had no significantly negative influence on the outcome of WT1-mutated patients (EFS HR: 1.605[0.886-2.906], $P=0.118$; OS HR: $1.748[0.870-$ 3.514], $P=0.117)$.

Similar results were found in the subgroup of cytogenetically normal AML patients according to the combined WT1 mutations and positive FLT3/ITD status (Figure S2). Of note, the survival curves showed that there were no significant differences between WT1-mutated patients with FLT3/ITDpositive $(n=17)$ and FLT3/ITD negative $(n=6)$, in terms of 5- year EFS $(14.1 \pm 9.0 \%$ vs $16.7 \pm 15.2 \% ; P=0.584)$ and $\mathrm{OS}(34.5 \pm$ $12.3 \%$ vs $33.3 \pm 19.2 \% ; P=0.665)$.

\section{The Effect of SCT in Patients With WT1 Mutations}

As shown in Table 1, there was no significant difference in the proportion of HSCT in WT1-mutated group and WT1 wild-type group ( $15.6 \%$ vs $16.2 \%, P=0.906)$. The survival analysis, after HSCT stratification, showed that for WT1-mutated pediatric AML patients, HSCT conferred a favorable prognostic impact with a trend of better 5-year EFS ( $42.9 \pm 18.7 \%$ vs $22.3 \pm 7.0 \%$ for chemotherapy-only; $P=0.316)$ and $O S(57.1 \pm 18.7 \%$ vs $43.6 \pm 8.2 \%$ for chemotherapyonly; $P=0.483$ ) (Figures 3A, B).

To further evaluate the role of HSCT in the patients with cooccurring WT1 and FLT3/ITD mutations, we explored the impact of HSCT on those patients. As shown in Figures 3C, D, for AML

\section{A}

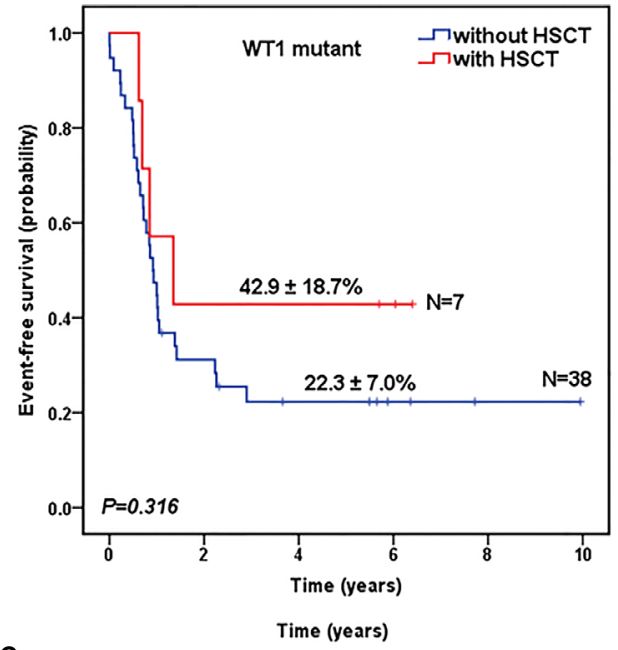

C

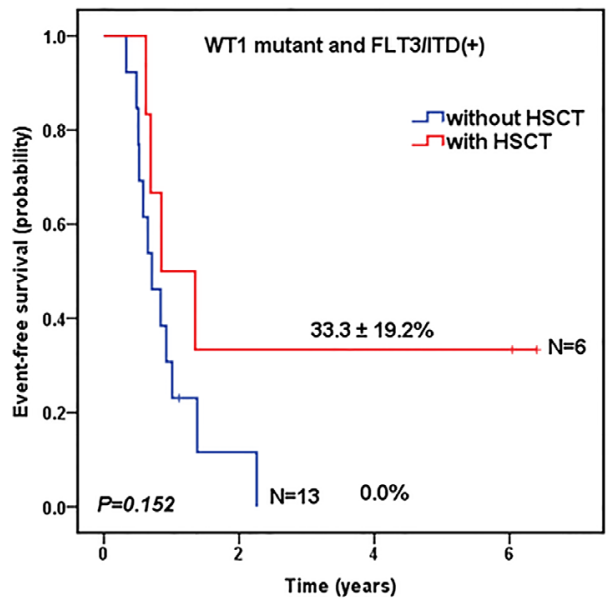

B
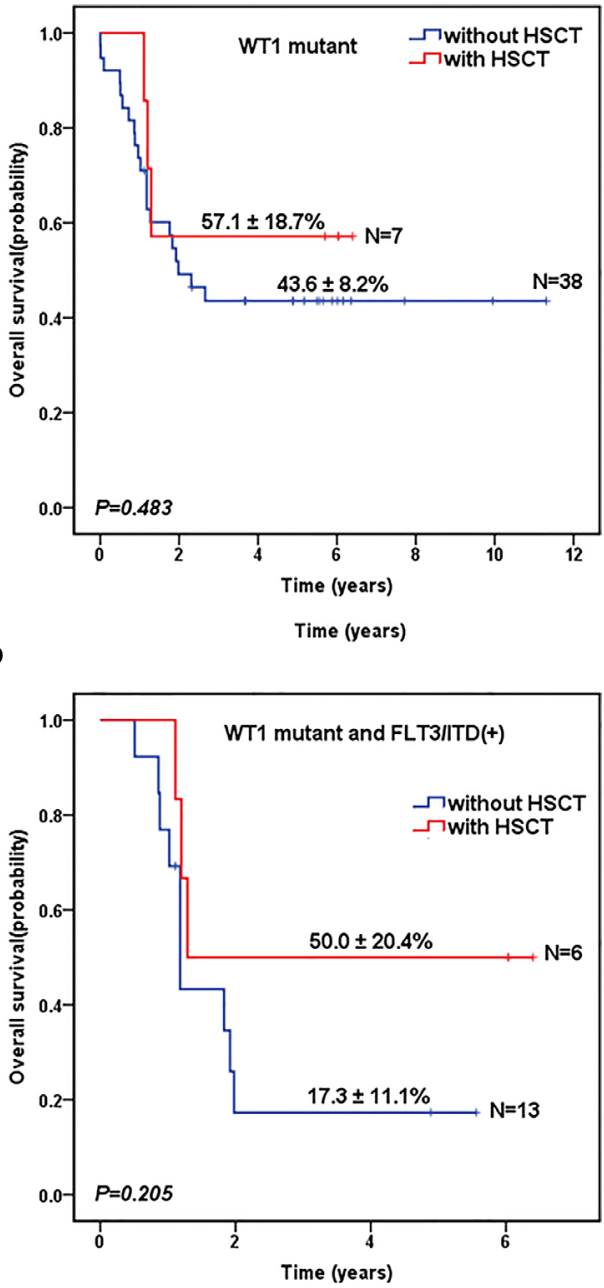

FIGURE 3 | Survival curves of pediatric AML patients according to WT1 mutations and hematopoietic stem cell transplantation (HSCT) status. Probability of EFS (A) and OS (B) for patients with WT1 mutations according to HSCT status, respectively. Probability of EFS (C) and OS (D) for patients with WT1 mutations and FLT3/ ITD positive according to HSCT status, respectively. 
patients with both WT1 mutations and positive FLT3/ITD, 5-year EFS $(33.3 \pm 19.2 \%)$ and OS $(50.0 \pm 20.4 \%)$ were higher in children with HSCT than those with chemotherapy-only (EFS: $0.0 \pm 0.0 \%$, $P=0.152$; OS: $17.3 \pm 11.1 \%, P=0.205)$, respectively, although the differences between the two groups were not statistically significant.

\section{Multivariate Analysis of Prognostic Factors}

Cox regression analyses were then performed to evaluate WT1 mutation status as a predictor of EFS and OS alongside other prognostic factors: age (utilizing 10 years of age as the cutoff value), white blood cell count at diagnosis (utilizing $50 \times 10^{9 /} \mathrm{L}$ as the cutoff value), high risk, standard risk, and HSCT. We identified WT1 mutations as an independent prognostic factor for both EFS and OS in pediatric patients with AML (Table 3). WT1 mutations were significantly associated with inferior EFS (HR: 1.910, 95\% CI: 1.297-2.812, $P=0.001$ ) and OS (HR: 1.709, 95\% CI: $1.090-2.679, P=0.020$ ). Additionally, age (older than 10 years), white blood cell count greater than $50 \times 10^{9} / \mathrm{L}$ at first diagnosis, high-risk and standard-risk were significantly related to poor EFS and OS, while HSCT was related to better survival prognosis (HR: 0.431, 95\% CI: $0.313-0.593, P<0.001$ ) and OS (HR: 0.594, 95\% CI: 0.419-0.843, $P=0.004$ ).

\section{DISCUSSION}

The TARGET program is a collaborative COG-national cancer institute (NCI) project aiming to comprehensively characterize the mutational, transcriptional, and epigenetic landscapes of a large, well-annotated cohort of pediatric cancer (20). Using this large cohort of subjects, we were able to investigate the clinical implication of WT1 mutations in pediatric AML. Our findings showed that the frequency of WT1 mutations was $6.7 \%$ among these 870 pediatric AML patients. This result was similar to the adult AML studies. In a large cohort of adult AML study, the frequency of WT1 mutations among 3157 patients was reported to be $5.5 \%$ (21). Next, we found that WT1 mutations were

TABLE 3 | Cox regression analysis of WT1 mutations and other prognostic factors.

\begin{tabular}{llcc}
\hline Outcome & \multicolumn{1}{c}{ Variable } & Hazard ratio $(\mathbf{9 5} \% \mathbf{C l})$ & P-value \\
\hline EFS & WT1 & $1.910(1.297-2.812)$ & 0.001 \\
& High risk & $3.136(2.235-4.400)$ & $<0.001$ \\
& Standard risk & $2.581(2.207-3.286)$ & $<0.001$ \\
& HSCT & $0.431(0.313-0.593)$ & $<0.001$ \\
& Age $>10$ years & $1.300(1.053-1.607)$ & 0.015 \\
& WBC $>50 \times 10^{9} / \mathrm{L}$ & $1.499(1.220-1.841)$ & $<0.001$ \\
OS & WT1 & $1.709(1.090-2.679)$ & 0.02 \\
& High risk & $3.991(2.653-6.004)$ & $<0.001$ \\
& Standard risk & $3.413(2.494-4.670)$ & $<0.001$ \\
& HSCT & $0.594(0.419-0.843)$ & 0.004 \\
& Age $>10$ years & $1.496(1.158-1.933)$ & 0.002 \\
& WBC $>50 \times 10^{9} / \mathrm{L}$ & $1.307(1.018-1.677)$ & 0.036 \\
\hline
\end{tabular}

Cl, confidence interval; EFS, event-free survival; HSCT, hematopoietic stem cell transplantation; OS, overall survival; WBC, white blood cell count. significantly associated with FAB subtypes of M4, with high white blood cell counts at first diagnosis, normal cytogenetics, and FLT3/ITD mutations. However, no association was found between WT1 mutations and CEBPA mutations. These results were different from some of the other studies. For instance, a report by $\mathrm{Ho}$ et al. (14) also found that WT1 mutations were related to normal cytogenetics and FLT3/ITD mutations, but they found no correlation between WT1 mutations and white blood cell counts or M4 subtype. A pediatric AML report by Hollink et al. (13) showed that WT1 mutations clustered significantly in the subgroup with normal cytogenetics and were associated with FLT3/ITD and CEBPA mutations.

The prognostic impact of WT1 mutations has not been clarified in pediatric AML. In our study, we found that patients with WT1 mutations had lower CR induction rates, worse EFS and OS rates in comparison to patients without WT1 mutations. Patients with both WT1 and FLT3/ITD mutations had a dismal prognosis. The multivariate analysis showed that WT1 mutations were an independent adverse impact factor. These results are consistent with findings by Hollink et al. (13), though they found the $\mathrm{CR}$ induction rates did not differ significantly between patients with WT1-mutated and WT1 wild-type AML. A report from the French study group confirmed that WT1 mutations were an independent prognostic factor for pediatric AML (22). However, a report from the Japanese study group showed that WT1 mutations were related to a poor prognosis in patients with normal cytogenetics, excluding those with FLT3/ ITD and those younger than 3 years (23). By contrast, a report from the Nordic Society of Pediatric Hematology and Oncology (NOPHO) revealed that no significant correlation with survival was seen for WT1 mutations (24). Notably, they found that patients with WT1 mutations but negative FLT3/ITD had a superior EFS compared with patients with WT1 wildtype with or without concurrent FLT3/ITD (24). In adult studies, the presence of WT1 mutation has been found to be associated with poor clinical outcomes of AML patients in some but not all studies. In the studies from Cancer and Leukemia Group B (9) and Hou et al. (10), WT1 mutations were correlated with a poor prognosis in AML patients. However, in the study from the German-Austrian Study Group (11), WT1 mutation as a single molecular marker did not seem to impact the patient outcomes. These conflicting results may be due to the differences in sample size, exon of WT1 mutations, and variable treatment protocols across studies. It has been reported that the negative impact of WT1 mutations may be overcome by the use of repetitive cycles of high-dose cytarabine, especially in the subgroup of patients with negative FLT3/ITD genotype (11).

The mechanism of WT1 mutations in leukemogenesis remains elusive. Several different WT1 mutations have been described in AML, which occur primarily in exons 1, 7, and 9. WT1 mutations may result in the loss of DNA binding ability due to loss of the zinc-finger domain or result in loss of expression of the WT1 protein altogether (25-27). WT1 mutations fail to properly direct the ten-eleven translocation-2 to its target sites, either by disruption of the interaction itself or by failing to bind to DNA $(28,29)$. Recently, Pronier et al. (30) 
have found that WT1 heterozygous loss enhances stem cell selfrenewal, WT1 depletion cooperates with FLT3/ITD mutation to induce fully penetrant AML. Mutational analysis of a large cohort of AML cases revealed that WT1 may play an important role in the epigenetic pathway $(31,32)$. Given the epigenetic alterations catalogued in WT1 mutant, epigenetictargeted therapy has been explored as a potential mechanism to deal with this subgroup of leukemia (33). Recently, Sinha et al. (34) have found that mutant WT1 is associated with DNA hypermethylation of polycomb repressor complex 2 targets in AML, and inhibitor of enhancer of zeste homolog 2 (EZH2) may be helpful in this AML subtype.

Alternately, HSCT is one of the most effective treatments for AML. However, it is unknown whether WT1-mutated patients will benefit from HSCT. Our studies showed that compared to chemotherapy alone, HSCT tended to improve the prognoses of WT1-mutated patients, and for patients with both WT1 and FLT3/ITD mutations as well. These results are in agreement with a previous pediatric AML report (14). Recently, Eisfeld et al. (12) have found that co-occurrence of WT1 and NPM1 mutations confers especially poor outcomes in a large cohort of 863 adult AML. They proposed that mutated WT1 co-occurrence with mutated NPM1 would be an adverse marker for risk stratification, indicating patients with both WT1 and NPM1 mutations might be considered for HSCT. However, since NPM1 mutation is relatively rare in children, we could not draw a firm conclusion on this topic due to the small number of patients with both WT1 and NPM1 mutations. Thus, whether WT1 mutation is an indication for HSCT in pediatric AML requires further investigation.

There were several limitations to our study. Firstly, since different WT1 mutations may affect its functions on DNA binding or protein interaction differentially, the details of WT1 mutants can be important to the clinical outcome of AML patients with these mutants. However, the information on the specific mutations of in WT1 is not provided in the TARGET dataset, therefore, we can't perform further analysis. Secondly, though this is a large pediatric AML cohort study, the sample size is still relatively small in the subgroups of patients with WT1 mutations. We cannot rule out the contribution of FLT3/ITD co-occurrence towards the prognosis. Thirdly, our findings showed that WT1 mutations were associated with poor clinical outcomes, and WT1-mutated patients might benefit from HSCT. These results suggested that WT1 mutations could be used as predictive factors and linked to a specific clinical management plan. However, due to the limitations associated with the TARGET dataset as mentioned above, and the retrospective analysis nature of our study, a large multicentric prospective future study could be of value to further address the prognostic significance of WT1 mutations in AML.

In summary, we analyzed the clinical implication of WT1 mutations in a large pediatric AML cohort. Our findings showed that WT1 mutations are independent poor prognostic factors in pediatric AML. Patients with cooccurring WT1 and FLT3/ITD mutations had a dismal prognosis. Moreover, HSCT might be an effective strategy for patients with WT1 mutations. These results have important implications and might contribute to the refining risk stratification of pediatric AML.

\section{DATA AVAILABILITY STATEMENT}

The original contributions presented in the study are included in the article/Supplementary Material, further inquiries can be directed to the corresponding authors.

\section{ETHICS STATEMENT}

The studies involving human participants were reviewed and approved by TARGET Publications Committee. Written informed consent to participate in this study was provided by the participants' legal guardian/next of kin.

\section{AUTHOR CONTRIBUTIONS}

LC and L-HX participated in project design, data collection, analysis, interpretation and manuscript drafting. YW participated in data interpretation and manuscript drafting. W-JW participated in data collection and analysis. D-HZ and J-PF participated in project design, data interpretation and manuscript drafting. SM participated in manuscript editing. All authors contributed to the article and approved the submitted version.

\section{FUNDING}

This work was supported by the Natural Science Foundation of Guangdong Province, China (2018A030313680 to L-HX), Guangdong Basic and Applied Basic Research Foundation (2020A1515010312 to L-HX), Science and Technology Program of Guangzhou City, China (201803010032 to J-PF), Beijing Bethune Charitable Foundation (SCE111DS to J-PF), Xiu Research Fund (to LC).

\section{ACKNOWLEDGMENTS}

We would like to thank all study participants and the TARGET group for making these data publicly available.

\section{SUPPLEMENTARY MATERIAL}

The Supplementary Material for this article can be found online at: https://www.frontiersin.org/articles/10.3389/fonc.2021. 632094/full\#supplementary-material 


\section{REFERENCES}

1. Creutzig U, van den Heuvel-Eibrink MM, Gibson B, Dworzak MN, Adachi S, de Bont E, et al. Diagnosis and management of acute myeloid leukemia in children and adolescents: recommendations from an international expert panel. Blood (2012) 120:3187-205. doi: 10.1182/blood-2012-03-362608

2. de Rooij JD, Zwaan CM, van den Heuvel-Eibrink M. Pediatric AML: From Biology to Clinical Management. J Clin Med (2015) 4:127-49. doi: 10.3390/ jcm 4010127

3. Conneely SE, Rau RE. The genomics of acute myeloid leukemia in children. Cancer Metastasis Rev (2020) 39:189-209. doi: 10.1007/s10555-020-09846-1

4. Scharnhorst V, van der Eb AJ, Jochemsen AG. WT1 proteins: functions in growth and differentiation. Gene (2001) 273:141-61. doi: 10.1016/s0378-1119 (01)00593-5

5. Yang L, Han Y, Suarez Saiz F, Minden MD. A tumor suppressor and oncogene: the WT1 story. Leukemia (2007) 21:868-76. doi: 10.1038/ sj.leu.2404624

6. Hohenstein P, Hastie ND. The many facets of the Wilms' tumour gene, WT1. Hum Mol Genet (2006) 15:R196-201. doi: 10.1093/hmg/ddl196

7. Hastie ND. Wilms' tumour 1 (WT1) in development, homeostasis and disease. Development (2017) 144:2862-72. doi: 10.1242/dev.153163

8. Owen C, Fitzgibbon J, Paschka P. The clinical relevance of Wilms Tumour 1 (WT1) gene mutations in acute leukaemia. Hematol Oncol (2010) 28:13-9. doi: 10.1002/hon.931

9. Paschka P, Marcucci G, Ruppert AS, Whitman SP, Mrozek K, Maharry K, et al. Wilms' tumor 1 gene mutations independently predict poor outcome in adults with cytogenetically normal acute myeloid leukemia: a cancer and leukemia group B study. J Clin Oncol (2008) 26:4595-602. doi: 10.1200/ JCO.2007.15.2058

10. Hou HA, Huang TC, Lin LI, Liu CY, Chen CY, Chou WC, et al. WT1 mutation in 470 adult patients with acute myeloid leukemia: stability during disease evolution and implication of its incorporation into a survival scoring system. Blood (2010) 115:5222-31. doi: 10.1182/blood-2009-12-259390

11. Gaidzik VI, Schlenk RF, Moschny S, Becker A, Bullinger L, Corbacioglu A, et al. Prognostic impact of WT1 mutations in cytogenetically normal acute myeloid leukemia: a study of the German-Austrian AML Study Group. Blood (2009) 113:4505-11. doi: 10.1182/blood-2008-10-183392

12. Eisfeld AK, Kohlschmidt J, Mims A, Nicolet D, Walker CJ, Blachly JS, et al. Additional gene mutations may refine the 2017 European LeukemiaNet classification in adult patients with de novo acute myeloid leukemia aged $<60$ years. Leukemia (2020) 34:3215-27. doi: 10.1038/s41375-020-0872-3

13. Hollink IH, van den Heuvel-Eibrink MM, Zimmermann M, Balgobind BV, Arentsen-Peters ST, Alders M, et al. Clinical relevance of Wilms tumor 1 gene mutations in childhood acute myeloid leukemia. Blood (2009) 113:5951-60. doi: 10.1182/blood-2008-09-177949

14. Ho PA, Zeng R, Alonzo TA, Gerbing RB, Miller KL, Pollard JA, et al. Prevalence and prognostic implications of WT1 mutations in pediatric acute myeloid leukemia (AML): a report from the Children's Oncology Group. Blood (2010) 116:702-10. doi: 10.1182/blood-2010-02-268953

15. Niktoreh N, Walter C, Zimmermann M, von Neuhoff C, von Neuhoff N, Rasche M, et al. Mutated WT1, FLT3-ITD, and NUP98-NSD1 Fusion in Various Combinations Define a Poor Prognostic Group in Pediatric Acute Myeloid Leukemia. J Oncol (2019) 2019:1609128. doi: 10.1155/2019/1609128

16. Meshinchi S, Alonzo TA, Stirewalt DL, Zwaan M, Zimmerman M, Reinhardt D, et al. Clinical implications of FLT3 mutations in pediatric AML. Blood (2006) 108:3654-61. doi: 10.1182/blood-2006-03-009233

17. Brown P, McIntyre E, Rau R, Meshinchi S, Lacayo N, Dahl G, et al. The incidence and clinical significance of nucleophosmin mutations in childhood AML. Blood (2007) 110:979-85. doi: 10.1182/blood-2007-02-076604

18. Ho PA, Alonzo TA, Gerbing RB, Pollard J, Stirewalt DL, Hurwitz C, et al. Prevalence and prognostic implications of CEBPA mutations in pediatric acute myeloid leukemia (AML): a report from the Children's Oncology Group. Blood (2009) 113:6558-66. doi: 10.1182/blood-2008-10-184747

19. Vujkovic M, Attiyeh EF, Ries RE, Goodman EK, Ding Y, Kavcic M, et al. Genomic architecture and treatment outcome in pediatric acute myeloid leukemia: a Children's Oncology Group report. Blood (2017) 129:3051-8. doi: 10.1182/blood-2017-03-772384

20. Bolouri H, Farrar JE, Triche TJr, Ries RE, Lim EL, Alonzo TA, et al. The molecular landscape of pediatric acute myeloid leukemia reveals recurrent structural alterations and age-specific mutational interactions. Nat Med (2018) 24:103-12. doi: 10.1038/nm.4439

21. Krauth MT, Alpermann T, Bacher U, Eder C, Dicker F, Ulke M, et al. WT1 mutations are secondary events in AML, show varying frequencies and impact on prognosis between genetic subgroups. Leukemia (2015) 29:660-7. doi: $10.1038 /$ leu.2014.243

22. Marceau-Renaut A, Duployez N, Ducourneau B, Labopin M, Petit A, Rousseau A, et al. Molecular Profiling Defines Distinct Prognostic Subgroups in Childhood AML: A Report From the French ELAM02 Study Group. Hemasphere (2018) 2:e31. doi: 10.1097/HS9.0000000000000031

23. Sano H, Shimada A, Tabuchi K, Taki T, Murata C, Park MJ, et al. WT1 mutation in pediatric patients with acute myeloid leukemia: a report from the Japanese Childhood AML Cooperative Study Group. Int J Hematol (2013) 98 (4):437-45. doi: 10.1007/s12185-013-1409-6

24. Staffas A, Kanduri M, Hovland R, Rosenquist R, Ommen HB, Abrahamsson J, et al. Presence of FLT3-ITD and high BAALC expression are independent prognostic markers in childhood acute myeloid leukemia. Blood (2011) 118:5905-13. doi: 10.1182/blood-2011-05-353185

25. King-Underwood L, Renshaw J, Pritchard-Jones K. Mutations in the Wilms' tumor gene WT1 in leukemias. Blood (1996) 87:2171-9. doi: 10.1182/ blood.V87.6.2171.bloodjournal8762171

26. Chen Z. The possible role and application of WT1 in human leukemia. Int $J$ Hematol (2001) 73:39-46. doi: 10.1007/BF02981901

27. Bielinska E, Matiakowska K, Haus O. Heterogeneity of human WT1 gene. Postepy Hig Med Dosw (Online) (2017) 71:595-601. doi: 10.5604/ 01.3001.0010.3840

28. Wang Y, Xiao M, Chen X, Chen L, Xu Y, Lv L, et al. WT1 recruits TET2 to regulate its target gene expression and suppress leukemia cell proliferation. Mol Cell (2015) 57:662-73. doi: 10.1016/j.molcel.2014.12.023

29. Rampal R, Figueroa ME. Wilms tumor 1 mutations in the pathogenesis of acute myeloid leukemia. Haematologica (2016) 101:672-9. doi: 10.3324/ haematol.2015.141796

30. Pronier E, Bowman RL, Ahn J, Glass J, Kandoth C, Merlinsky TR, et al. Genetic and epigenetic evolution as a contributor to WT1-mutant leukemogenesis. Blood (2018) 132:1265-78. doi: 10.1182/blood-2018-03837468

31. Patel JP, Gonen M, Figueroa ME, Fernandez H, Sun Z, Racevskis J, et al. Prognostic relevance of integrated genetic profiling in acute myeloid leukemia. N Engl J Med (2012) 366:1079-89. doi: 10.1056/NEJMoa1112304

32. Fernandez HF, Sun Z, Yao X, Litzow MR, Luger SM, Paietta EM, et al. Anthracycline dose intensification in acute myeloid leukemia. N Engl J Med (2009) 361:1249-59. doi: 10.1056/NEJMoa0904544

33. Panuzzo C, Signorino E, Calabrese C, Ali MS, Petiti J, Bracco E, et al. Landscape of Tumor Suppressor Mutations in Acute Myeloid Leukemia. J Clin Med (2020) 9(3):802. doi: 10.3390/jcm9030802

34. Sinha S, Thomas D, Yu L, Gentles AJ, Jung N, Corces-Zimmerman MR, et al. Mutant WT1 is associated with DNA hypermethylation of PRC2 targets in AML and responds to EZH2 inhibition. Blood (2015) 125:316-26. doi: 10.1182/blood-2014-03-566018

Conflict of Interest: The authors declare that the research was conducted in the absence of any commercial or financial relationships that could be construed as a potential conflict of interest.

Copyright $\odot 2021$ Wang, Weng, Zhou, Fang, Mishra, Chai and Xu. This is an openaccess article distributed under the terms of the Creative Commons Attribution License (CC BY). The use, distribution or reproduction in other forums is permitted, provided the original author(s) and the copyright owner(s) are credited and that the original publication in this journal is cited, in accordance with accepted academic practice. No use, distribution or reproduction is permitted which does not comply with these terms. 